\title{
Consensus in Innovation Contest Categorisation by means of Fuzzy Partitions
}

\author{
Albert Armisen ${ }^{\mathrm{a}}$, Germán Sánchez-Hernández ${ }^{\mathrm{a}, *}$, Ann Majchrzak ${ }^{\mathrm{a}, \mathrm{b}}$, Núria \\ Agell $^{\mathrm{a}}$ \\ ${ }^{a}$ Information Systems Department, ESADE Business School, Ramon Llull Universities, Av. \\ Pedralbes, 60-62, Barcelona, Spain \\ ${ }^{b}$ Data Science and Operations Department, Marshall School of Business, University of \\ Southern California, Los Angeles, USA
}

\begin{abstract}
Consensus decision-making is fuzzy by nature, yet fuzzy consensus decisionmaking in a medium to large number of decisions is not widely used since it demands additional information that requires extra decision-maker effort. Consensus decision-making rests on properly measured agreement. This paper proposes a fuzzy measure of agreement through fuzzy kappa based on fuzzy partitions. These fuzzy partitions enable decision-makers to assess their decisions with a degree of confidence. A fuzzy partition is built for each decision-maker considering his/her confidence degrees when categorising a set of alternatives or solutions. This enables decision-makers to more easily capture the fuzzy nature of the decision. In addition, this paper presents a real-life experiment based on a innovation contest to show the feasibility of using confidence degrees in real-life applications compared to traditional consensus decision-making. The results suggest that the use of confidence degrees improves the level of agreement in the consensus decision-making process through fuzzy kappa coefficients, and it also improves the level of agreement in the consensus decision-making process. Keywords: Fuzzy partitions, Fuzzy sets, fuzzy kappa, Cohen's kappa, innovation contest, open innovation, consensus decision-making, crowdsourcing
\end{abstract}

\footnotetext{
${ }^{*}$ Corresponding author

Email addresses: albert.armisen@gmail.com (Albert Armisen), german.sanchez@esade.edu (Germán Sánchez-Hernández), majchrza@usc.edu (Ann Majchrzak), nuria.agell@esade.edu (Núria Agell)

Preprint submitted to Applied Soft Computing

March 13, 2015
}

(C) 2015. This manuscript version is made available under the Elsevier user license http://www.elsevier.com/open-access/userlicense/1.0/ 


\section{Introduction}

A key challenge for organisations is to maintain their competitiveness through innovation. Critical to innovation is the fuzzy-front end of the new product development process (Klink and Athaide, 2006). Idea generation and selection e most innovative ideas to pursue is at the core of this front-end process (Toubia, 2006). To increase the chances of identifying promising new products, processes, and strategic or improvement ideas, individuals from a large variety of sources are solicited for these ideas (e.g. users, employees, manufacturers, suppliers, regulators, government agencies, non-government organisations, and general members of society) (Chesbrough, 2006; Von Hippel, 1989).

These requests for innovative ideas are often part of what are referred to as innovation crowdsourcing events (Leimeister et al., 2009; Majchrzak and Malhotra, 2013, Surowiecki, 2005). In these events, an announcement is made by a sponsoring organisation to a variety of sources (via advertisements, postings on list servers, or invitations). The announcement describes the sponsoring organisation; the problem, issue, or question that the organisation is raising; information about the problem; a request for innovative ideas; a website where contributions can be made; and a timeframe (from three days to three months) during which the event will occur. Such events are increasingly used for soliciting new research \& development $(\mathrm{R} \& \mathrm{D})$ ideas as part of a paradigm shift referred to as open innovation (Chesbrough, 2006). Government (Administration, 2015) and non-government organisations (of the Red Cross, 2015), as well [as private sector companies (e.g., King and Lakhani, 2011, Malhotra and Majchrzak, 2014)) request ideas ranging from new business strategies (Malhotra

25 and Majchrzak, 2014) to how to provide water supplies (OpenIDEO, 2015). These innovation crowdsourcing events are known as innovation contests or community-based innovation contests (Bullinger et al., 2010, Leimeister et al. 2009, Majchrzak and Malhotra, 2013, Piller and Walcher, 2006).

In a successful innovation initiative, be it crowdsourced or more traditional 
methods of customer focus groups, a large number of new ideas are generated (Diehl and Stroebe, 1987, Neyer et al., 2009, Simonton, 1999). In fact, if the innovation initiative is very successful, it may generate so many ideas that the selection of the most promising ones becomes very difficult or costly (Santos and Spann, 2011). An innovation initiative requires identifying both innovative

35 ideas and interactions (e.g. posts in an innovation contest). The first step is to select a set of categorised innovative ideas from a large number of ideas found in one or more participant interactions. The chief innovation officers (CIOs) select innovative ideas using a criteria including novelty, competitive advantage, feasibility, and fit with existing competencies (Cooper and De Brentani, 1984).

40 The second step is to identify posts based on the innovative ideas selected to properly motivate participants (Füller et al. 2012). This identification is, in general, done by a group of decision-makers or R\&D managers who categorise posts as matching the set of CIO-selected ideas (Lamastra, 2009). An alternative approach is for CIOs to select the innovative ideas and posts: however, this

${ }_{45}$ approach requires more time from CIOs (and whose time is scarce) (Toubia, 2006).

घ Categorising posts based on innovative ideas is inherently subjective (Piller and Walcher, 2006) since "the process of decision-making, in particular of a group type, is centered on humans, coming with their inherent subjectivity, im${ }_{50}$ precision and vagueness in the articulation of opinions" (Herrera-Viedma et al. 2014 p. 5). In the case of an innovation contest, the use of confidence degrees (i.e. a parameter that captures the certainty of the decision-maker or $\mathrm{R} \& \mathrm{D}$ manager) enables us to capture the fuzzy nature of the process and so make it closer to reality. Despite an increase in effort by decision-makers being required when using confidence degrees, their use enables harnessing information about uncertainty from each decision-maker in order to better obtain a soft consensus. Confidence degrees are obtained by means of a set of linguistic labels. The use of these linguistic labels enables us to generate a fuzzy partition per decision-maker. Finally, the coincidence matrix is assessed to determine the ${ }_{60}$ shared memberships of each post. The soft consensus degree is then computed 
using a fuzzy kappa (Dou et al., 2007).

The paper is organised as follows. Section 2 establishes the theoretical framework regarding consensus-decision-making and fuzzy partitions. Section 3 defines the framework and associated methodology used to translate a crisp 65 partition into a fuzzy one by exploiting the degrees of confidence of the decisionmakers. In Section 4 a real-case application of the methodology presented is detailed along with research findings and results. Section 5 discuses the implication of selecting different parameters in our methodology. Finally, the conclusions and directions for further research are explained in Section 6 .

\section{Theoretical framework}

In this section, firstly some theoretical background information on the consensus process is presented; secondly, an overview is provided on the use of fuzzy partitions for building a set of fuzzy indices (classically used to compare a given partition with an a priori structure, or just another partition) are anal-

75 ysed to compare the fuzzy partitions obtained. Finally, these indices are used to measure agreement and extended for more than two decision-makers.

\subsection{Consensus process}

Group decision-making (GDM) is a problem-solving activity involving groups of decision-makers that produces a final choice based on the selection among several possibilities (Edwards, 1977). Consensus process is a step considered in some types of GDM technique that focuses on obtaining the maximum agreement between a set of decision-makers (Herrera-Viedma et al., 2007, 2002). In general, GDM requires the majority of individuals to approve a choice, and that the minority agrees with the final choice (Herrera-Viedma et al., 2002, Kaner, 85 2014). Other group decision-making techniques involve voting-based methods (Ayad and Kamel, 2010), or structured techniques consisting of several rounds using an anonymous summary known as Delphi (Linstone et al., 1975).

Consensus decision-making techniques seek to: 1) empower all participants in the decision process; 2) listen to other participants; 3) challenge the other 
perspective until reaching a consensus; 4) integrate different viewpoints from other participants; 5) provide the mechanics to work on the dynamics that are not rational (such as emotions and intuitions); and 6) allocate different times to each decision if need be (Bressen, 2007). These properties are better fulfilled when using confidence degrees as a feedback mechanism since this enables the (Edwards, 1977).

Traditional approaches to consensus decision-making that do not use confidence degrees are widely used when the subjective categorisation of information based on subjective criteria (such as novelty) is needed (Saldaña, 2012). This but a confidence degree for each assigned category) in a vague, complex, process that might introduce possible inconsistencies among the decision-makers (Martínez and Montero, 2007). Some sets of consensus decision-making techniques such as multi-attribute utility analysis use confidence degrees to capture 120

subjectiveness is handled by an inter-rater reliability assessment of the classifications assigned to the data, where two or more decision-makers analyse the data, and make various predefined categorisations (Gwet, 2014). A good categorisation that overcomes this subjectivity weakness is defined when the decisionmakers have an agreement that is higher than a specific threshold (Landis and Koch, 1977). Traditional approaches assume that each decision-maker has the same level of decision-making certainty, and so introducing unnecessary error into the categorising by assuming that each decision-maker is absolutely certain about the categories assigned. For example, two decision-makers seeking to categorise emotional intensity in an idea may not be absolutely certain.

Decision-making in a fuzzy environment is a decision process in which the goals, constraints, or the system under control are fuzzy by nature (Bellman and Zadeh, 1970). However, consensus decision-making for medium to large datasets has not adopted the use of fuzzy logic when categorising alternatives since adoption demands an additional decision-maker effort (e.g. a post to an online discussion thread would require not only the categorisation of a post to a category, the different decision-maker preferences (Torrance et al., 1982). Multi-attribute 
utility analysis has been extended to fuzzy environments using different coefficients (Chen, 2000, Chiclana et al. 2013). This paper proposes capturing and using in posterior discussion one confidence degree per decision instead of multiple confidence degrees.

Capturing one confidence degree simplifies the decision-making process by capturing the fuzzy nature of the decision, while adding a minimal additional effort for the decision-makers (e.g. a post with ten categories requires one confidence degree instead of ten confidence degrees). The use of these confidence degrees helps to overcome the uncertainty management challenge (Martínez and

130 Montero, 2007) by enhancing decision-makers with additional information to assess their own decision-maker certainty through a degree of confidence. Moreover, these confidence degrees enable capturing intuition, or a viewpoint that is worth exploring if there is disagreement, by setting a low confidence degree (Bressen, 2007). Thus, the fuzzy approach improves the good properties of consensus decision-making, while enabling normalising to reflect a decision-maker's personality with regard to confidence (i.e. overconfidence, confident, or underconfidence). This is known as a soft consensus approach (Cabrerizo et al. 2009 , 2010, Herrera-Viedma et al., 2005, 2007).

\subsection{Fuzzy partitions}

Within the framework of fuzzy sets introduced by Zadeh in 1965, a fuzzy set is characterised by a membership function that associates each element with a real number in the interval $[0,1]$. A fuzzy partition of a set is then defined by giving the degree of membership or adequacy of each individual to each considered category (classes or clusters). The formal description of a fuzzy partition was introduced by Ruspini (1969). A fuzzy partition of a given set $X=\left\{x_{1}, x_{2}, \ldots, x_{N}\right\}$ into $K$ clusters $C_{k}, k \in\{1, \ldots, K\}$, is defined by a set of values $\mu_{i k}$, in which each value indicates the membership degree of the alternative $i$ to the category $k$ and satisfies the following conditions:

$$
0 \leq \mu_{i k} \leq 1, \forall i, k \text { and } \forall k \exists i \text { such that } \mu_{i k}>0 .
$$


In general, a fuzzy partition is expressed by a matrix $F_{X}=\left(\mu_{i k}\right)$, where in each row, the marginal membership degree of the alternative $x_{i}$ to the category $C_{k}$ is represented. In addition, when the constraint $\sum_{k=1}^{K} \mu_{i k}=1, \forall i$ is satisfied, the fuzzy partition is termed a fuzzy probabilistic partition. Note that in the extreme case in which $\mu_{i k} \in\{0,1\} \forall i, k$, a crisp partition is obtained. Thus, fuzzy probabilistic partitions can be considered as a generalisation of crisp par5 titions, in the same way than fuzzy sets are considered as a generalisation of crisp sets.

To define a fuzzy degree of compatibility or similarity between fuzzy partitions, t-norms and t-conorms (denoted with a superscript '*') have extended intersection and union operators to fuzzy sets theory (Dubois and Prade, 2004).

160 The following t-norms and t-conorms are broadly used:

- MinMax:

- Min: $M\left(y_{1}, \ldots, y_{n}\right)=\min \left\{y_{1}, \ldots, y_{n}\right\}$

- Max: $M^{*}\left(y_{1}, \ldots, y_{n}\right)=\max \left\{y_{1}, \ldots, y_{n}\right\}$

- Probabilistic product:

165

$$
\begin{aligned}
& -\prod\left(y_{1}, \ldots, y_{n}\right)=y_{1} \cdot \ldots \cdot y_{n} \\
& -\prod^{*}\left(y_{1}, \ldots, y_{n}\right)=1-\prod_{i=1}^{n}\left(1-y_{i}\right)
\end{aligned}
$$

- Lukasiewicz:

$$
\begin{aligned}
& -W\left(y_{1}, \ldots, y_{n}\right)=\max \left\{1-n+\sum_{i=1}^{n} y_{i}, 0\right\} \\
& -W^{*}\left(y_{1}, \ldots, y_{n}\right)=\min \left\{\sum_{i=1}^{n} y_{i}, 1\right\}
\end{aligned}
$$

\subsection{Indices to compare crisp partitions}

The most classic indices to compare two partitions are described below. The Rand index (Rand, 1971), and most of the following indices, are based on pairwise comparisons of data alternatives. The original crisp version of these indices handles two crisp partition matrices $\left(P_{X}\right.$ and $\left.Q_{X}\right)$ of the same set of alternatives 
$X$. Both partitions can "categorise" data alternatives into a different number of clusters. The following sets should be defined to introduce these indices:

- $S_{1}$ is the subset of tuples $\{x, y\} \in X$ paired both in $P_{X}$ and in $Q_{X}$;

- $S_{2}$ is the subset of tuples $\{x, y\} \in X$ paired in $P_{X}$ but not paired in $Q_{X}$;

- $S_{3}$ is the subset of tuples $\{x, y\} \in X$ not paired in $P_{X}$ but paired in $Q_{X}$;

- $S_{4}$ is the subset of tuples $\{x, y\} \in X$ neither paired in $P_{X}$ nor in $Q_{X}$.

Let the following numbers be the cardinal of the previously defined sets:

$$
a=\left|S_{1}\right|, b=\left|S_{2}\right|, c=\left|S_{3}\right|, d=\left|S_{4}\right| .
$$

Note that $\left\{S_{1}, S_{2}, S_{3}, S_{4}\right\}$ is a partition of the set consisting of the subsets of two elements of $X$ and that $a+b+c+d=|X|$. On the one hand, $S_{1} \cup S_{4}$ are the concordant pairs between $P_{X}$ and $Q_{X}$ (agreements), while on the other hand $S_{2} \cup S_{3}$ are the discordant pairs (disagreements). Bearing this in mind, the Rand index is defined as the ratio between the concordant pairs and the total number of pairs. One of the main criticisms against the Rand index is that it is not corrected by chance, that is to say, its expected value is not zero when comparing two random partitions (Cohen, 1968). The adjusted Rand index is corrected by assuming that the number of clusters to be compared is the same in both partitions (Hubert and Arabie, 1985).

Another common criticism of the Rand index is that it gives the same importance to both agreement terms $a$ and $d$, thus making no difference between pairs of alternatives that are joined or separated in both partitions. Moreover, term $d$ may dominate the other terms $a, b$ and $c$. The removal of this term $d$ results in the so-called Jaccard coefficient (Halkidi et al., 2001). It can be interpreted as the proportion of good pairs with respect to non-neutral pairs. The absense of term $d$ is also exhibited in the so-called Fowlkes-Mallows index (Fowlkes and Mallows, 1983), Minkowski measure (Jiang et al., 2004) and the $200 \omega_{\tau}$ statistic (Jain et al., 1988). 
The main concern of these related indices is that they do not perform properly when the clusters to be analysed are imbalanced (i.e. the probability of one cluster vastly exceeds others) (Cohen, 1968). To solve this, Cohen's kappa coefficient measuring the similarity between two partitions (i.e. $P$ and $Q$ ) was defined as follows:

$$
\omega_{\kappa}(P, Q)=\kappa=\frac{p_{o}-p_{e}}{1-p_{e}},
$$

where $p_{o}$ is the relative coincidence observed between partition $P$ and $Q$ and $p_{e}$ is the hypothetical probability of chance coincidences, using the observed categorisations to compute the probabilities of each partition randomly choosing each category. If partitions completely match, then $\kappa=1$. However, if the categorisations differ completely, then $\kappa=0$. The computation of both terms $p_{o}$ and $p_{e}$ is detailed in Dou et al. (2007).

\subsection{Measuring Agreement: Indices to compare fuzzy partitions}

The design of suitable measures for comparing fuzzy partitions is an important topic of research in several multidisciplinary areas (Kukar, 2003: Osei215 Bryson, 2010) such as clustering. Some of the previously introduced indices in Subsection 2.3 have been extended into fuzzy versions: fuzzy Rand index and others (Campello, 2007) and fuzzy kappa index (Dou et al., 2007).

The extension of Cohen's kappa preserves the formula given in (2) for computing Cohen's kappa statistic by taking into account the fuzziness of the partition in the calculation of the proportions $p_{o}$ and $p_{e}$. The computation of such terms needs the selection of a t-norm in order to aggregate the membership degrees of a certain alternative to a category:

$$
\kappa_{\tau}=\frac{p_{o_{\tau}}-p_{e_{\tau}}}{1-p_{e_{\tau}}},
$$

where $\tau$ is a t-norm (see Subsection 2.2 and $p_{o_{\tau}}$ and $p_{e_{\tau}}$ stand for the observed and expected agreement, respectively, computed by employing the same t-norm ${ }_{225} \tau$. The values of $p_{o_{\tau}}$ and $p_{e_{\tau}}$ are defined by Dou et al. (2007) (see Subsection 
3.4 .

\section{A Fuzzy Methodology to Measure Agreement in Classification}

This section presents a fuzzy methodology to measure agreement based on confidence degrees and decision-maker preferences for two decision-makers. In Figure 1 the different steps of this methodology are highlighted. Initially, we present how to transform from a given crisp partition, in which each decisionmaker has categorised each alternative into a unique category (i.e. "3.1" on Figure 1 and in Subsection 3.1), to a fuzzy partition by taking into account the confidence degree stated by the decision-maker in each classification (i.e. "3.2" on Figure 1 and in Subsection 3.2. This section describes the use of fuzzy indices to define a fuzzy membership matrix: the coincidence matrix between decision-makers (i.e. "3.3" on Figure 1 and in Subsection 3.3). This coincidence matrix is used to measure the agreement or consensus degree (i.e. "3.4" on Figure 1 and in Subsection 3.4. This consensus degree, as well as the decisionmaker preferences and confidence degrees are used as feedback. For this reason, this framework can be classified as a feedback mechanism and consensus degree based on distance between the decision-makers according to the taxonomy given by Palomares et al. (2014). Finally, the methodology can be extended to more than two decision-makers following the steps defined in Subsection 3.5 .

Let us assume a decision-making problem in which two decision-makers $\mathcal{E}_{1}$ and $\mathcal{E}_{2}$ analyse a set of alternatives $X=\left\{x_{1}, \ldots, x_{i}, \ldots, x_{N}\right\}$ to classify or assign them on a set of categories or classes $C=\left\{c_{1}, \ldots, c_{k}, \ldots, c_{K}\right\}$ (i.e. each decision-maker $\mathcal{E}$ states his/her selections through his/her preferences associated to a crisp membership degree $\delta_{i k}^{\mathcal{E}} \in\{0,1\}, \forall i \in\{1, \ldots N\}$ and $\left.k \in\{1, \ldots K\}\right)$. In addition, decision-maker $\mathcal{E}$ associates with each alternative a confidence degree $D_{i}^{\mathcal{E}}, \forall i \in\{1, \ldots N\}$, to express the level of confidence that he/she has when assigning a category to this alternative. The values of this confidence degree are given by a linguistic ordinal term set $S=\left\{s_{0}, \ldots, s_{g}\right\}$ whose cardinality or granularity is $\# S=g+1$, being $D_{i}^{\mathcal{E}} \in S$. Therefore, an $N$-dimensional vector 


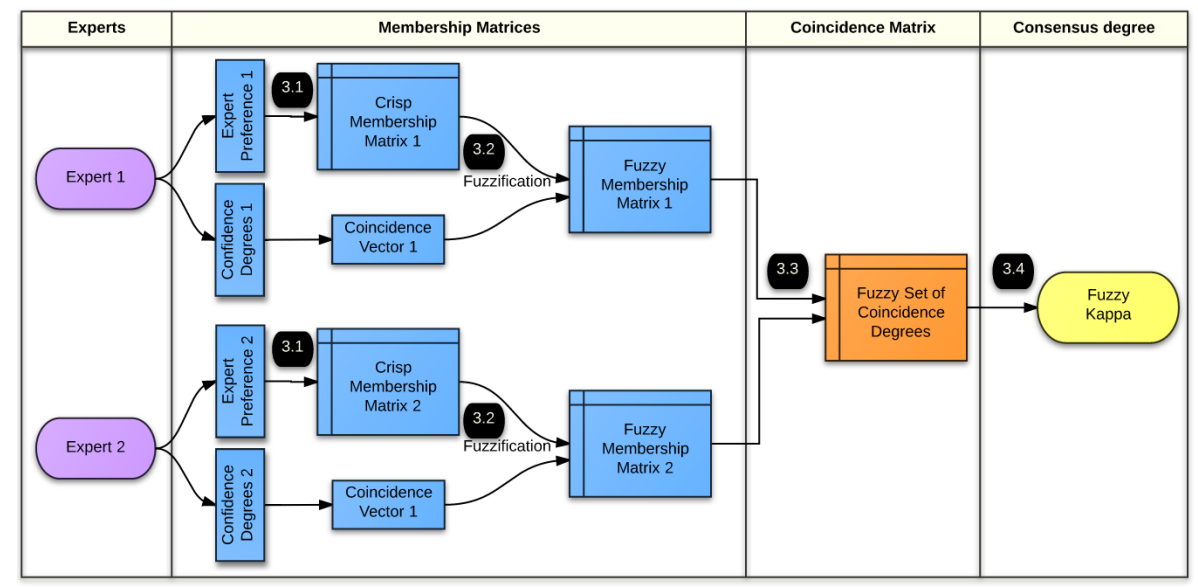

Figure 1: Scheme of the two-decision-maker soft consensus agreement proposed. Number indicates the section in which each step is detailed.

$D^{\mathcal{E}}=\left\{D_{1}^{\mathcal{E}}, \ldots, D_{N}^{\mathcal{E}}\right\}$ is associated with decision-maker $\mathcal{E}$

\subsection{Stating a crisp partition}

Algorithm 1 enables us to define, for each decision-maker $\mathcal{E}_{1}$ and $\mathcal{E}_{2}$, his/her crisp membership matrix according to the initial decision-maker preferences.

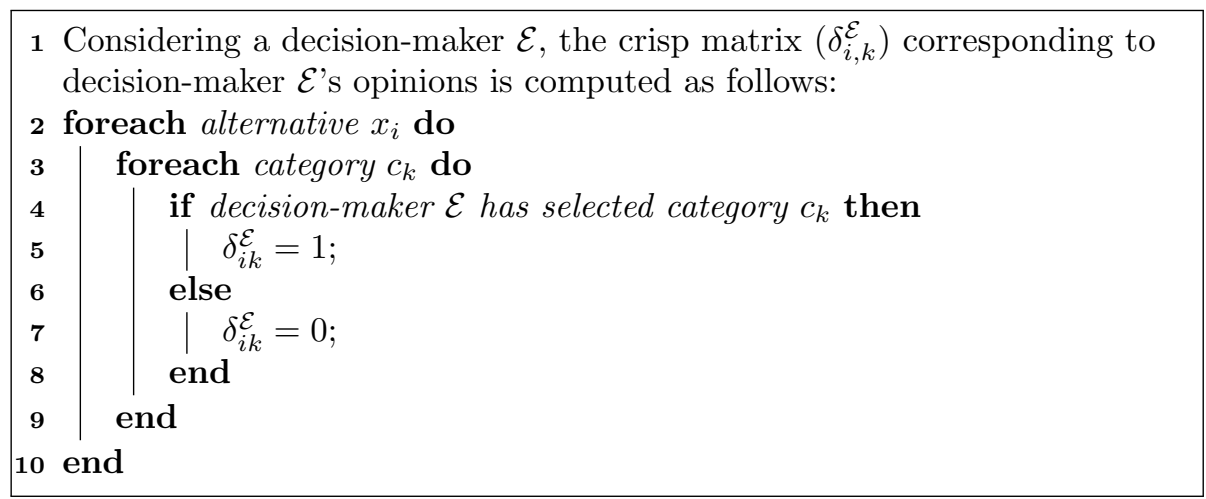

Algorithm 1: Defining the crisp partition

Example 1. Let us assume that two averagely confident decision-makers $\mathcal{E}=\left\{\mathcal{E}_{1}, \mathcal{E}_{2}\right\}$ provide their decision-maker preference on classifying a set of four alternatives $X=\left\{x_{1}, x_{2}, x_{3}, x_{4}\right\}$ in three categories $\mathcal{C}=\{A, B, C\}$. They 
use the linguistic term set $S=\{$ Strongly uncertain(0), Uncertain(1), Partially uncertain(2), Neither certain nor uncertain(3), Partially certain(4), Certain(5), Strongly certain $(6)\}$ to express their confidence, as depicted in Table 1.

Table 1: Example for decision-maker preferences and confidence degrees for three alternatives

\begin{tabular}{c|cc|cc} 
& \multicolumn{2}{|c|}{ Decision-maker \#1 } & \multicolumn{2}{c}{ Decision-maker \#2 } \\
& Preference & Confidence Degree & Preference & Confidence Degree \\
\hline$x_{1}$ & A & 3 & A & 5 \\
$x_{2}$ & C & 0 & C & 0 \\
$x_{3}$ & C & 2 & B & 5 \\
$x_{4}$ & A & 1 & C & 2
\end{tabular}

In this first step we transform this data to a crisp partition obtaining the results detailed in Table 2 .

Table 2: Transformation example for crisp partitions for the two decision-makers, four alternatives, and three categories

\begin{tabular}{|c|c|c|c|c|c|c|c|}
\hline & \multicolumn{3}{|c|}{ DM \#1 } & & \multicolumn{3}{|c|}{$\mathrm{DM} \# 2$} \\
\hline & A & B & $\mathrm{C}$ & & A & B & $\mathrm{C}$ \\
\hline$x_{1}$ & 1 & 0 & 0 & $x_{1}$ & 1 & 0 & 0 \\
\hline$x_{2}$ & 0 & 0 & 1 & $x_{2}$ & 0 & 0 & 1 \\
\hline$x_{3}$ & 0 & 0 & 1 & $x_{3}$ & 0 & 1 & 0 \\
\hline$x_{4}$ & 1 & 0 & 0 & $x_{3}$ & 0 & 0 & 1 \\
\hline
\end{tabular}

\subsection{Fuzzification}

To make use of the extra information provided by the $N$-dimensional vector $D^{\mathcal{E}}$ given by decision-maker $\mathcal{E}$, this subsection introduces a methodology to 270 translate the crisp partition defined by $\left(\delta_{i k}^{\mathcal{E}}\right)$ to a fuzzy one defined by $\left(\mu_{i k}^{\mathcal{E}}\right)$. This new fuzzy partition is defined from the confidence degree vector $D^{\mathcal{E}}$, and the crisp membership values, $\left(\delta_{i k}^{\mathcal{E}}\right)$, and satisfies the following conditions:

1. $\mu_{i k}^{\mathcal{E}} \in[0,1], \forall i, k$;

2. $\sum_{k=1}^{K} \mu_{i k}^{\mathcal{E}}=1, \forall i$;

275
3. $\mu_{i k}^{\mathcal{E}}=1 \Longleftrightarrow \delta_{i k}^{\mathcal{E}}=1$ and $D_{i}^{\mathcal{E}}=\max (S)=s_{g}$;
4. $\mu_{i k}^{\mathcal{E}}=\frac{1}{K} \Longleftrightarrow D_{i}^{\mathcal{E}}=\min (S)=s_{0}$; 
5. An interpolation function $f$ is considered to define $\mu_{i k}$ when $D_{i}^{\mathcal{E}} \neq s_{0}$ and $D_{i}^{\mathcal{E}} \neq s_{g}$.

Three different functions (i.e. linear, logarithmic, and exponential) have been considered to obtain the interpolation in condition \#5. Figure 2 displays on the ordinates axis the new membership degree of the category originally chosen for the alternative, according to the confidence degree shown in the abscesses axis.

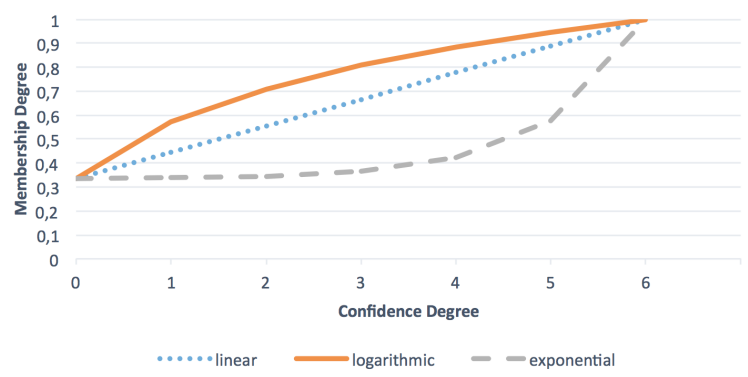

Figure 2: Example of the fuzzy membership degree of a category that had a crisp membership degree of 1. Logarithmic, linear and exponential functions are depicted by taking $K=3$ and $\# S=7$.

The fuzzification is a two-step process. The first step is to compute the maximum value for each alternative using one of the three interpolation functions introduced. Given an alternative $x_{i} i \in\{1, \ldots, N\}$ and that $k^{*} \in\{1, \ldots, K\}$ such that $\delta_{i k^{*} \mathcal{E}}=1$. Then, $\mu_{i k^{*}}$ is defined according to Table 3 .

\begin{tabular}{c|cc}
\multicolumn{3}{c}{ Table 3: Three different functions of fuzzification } \\
& $\mu_{i k^{*}}$ & Decision-maker assumption \\
\hline Linear & $\frac{D_{i}}{\# S-1} \cdot \frac{K-1}{K}+\frac{1}{K}$ & Average confidence \\
Exponential & $\frac{\log \left(1+D_{i}\right)}{\log (\# S)} \cdot \frac{K-1}{K}+\frac{1}{K}$ & Underconfident \\
Logarithmic & $\frac{e^{D_{i}}}{e^{\# S-1}} \cdot \frac{K-1}{K}+\frac{1}{K}$ & Overconfident
\end{tabular}

The second step is to compute the fuzzy membership degrees of the other categories, $\mu_{i \bar{k}^{*}}$. They are equally defined to satisfy condition \#2 as follows:

$$
\mu_{i k}=\frac{1-\mu_{i k^{*}}}{K-1}, \forall k \neq k^{*}
$$


The indices defined in Subsection 2.2 are usually used to measure the compatibility between two partitions of the same set of alternatives. Applying the methodology detailed in the previous Subsection 3.2, any crisp partition with an associated vector of degree of confidence can be easily transformed into a fuzzy partition. The process for fuzzifying opinions made by a decision-maker $\mathcal{E}$ on selecting from a set $C$ of $K$ alternatives is detailed in Algorithm 2 .

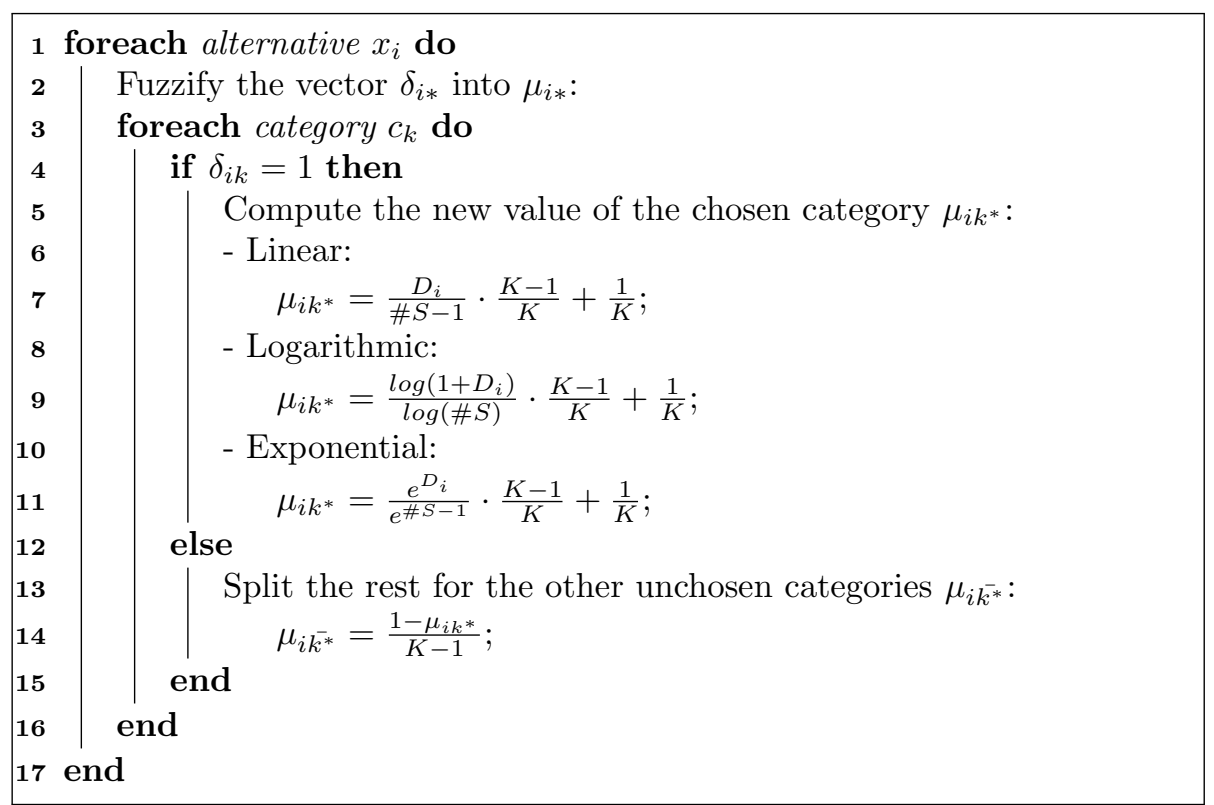

Algorithm 2: Fuzzification

Example 2. Using the previous example where the crisp partitions were stated, their fuzzification is done based on the confidence degrees and linear interpolation (i.e. given by the normal confidence of the decision-makers).

Let us take the membership degree stated decision-maker $\mathcal{E}_{1}$ regarding the first alternative and first category $\delta_{11}=1$, with confidence $D_{1}=3$. Given that ${ }_{300} K=3, \mu_{1 k^{*}}=\frac{D_{1}}{\# S-1} \cdot \frac{K-1}{K}+\frac{1}{K}=\frac{3}{7} \cdot \frac{3-1}{3}+\frac{1}{3}=0.667$. The others values $\mu_{1 k^{*}}=\frac{1-\mu_{1 k^{*}}}{K-1}=\frac{0.667}{3-1}=0.167$. Table 4 depicts the values obtained from this example. 
Table 4: Example of fuzzy partitions for two decision-makers in three alternatives

\begin{tabular}{l|cccc|cccc} 
& \multicolumn{2}{|c}{ Decision-maker \#1 } & & \multicolumn{3}{|c}{ Decision-maker \#2 } \\
& $\mathrm{A}$ & $\mathrm{B}$ & $\mathrm{C}$ & & & $\mathrm{A}$ & $\mathrm{B}$ & $\mathrm{C}$ \\
\hline$x_{1}$ & 0.667 & 0.167 & 0.167 & & $x_{1}$ & 0.889 & 0.056 & 0.056 \\
$x_{2}$ & 0.333 & 0.333 & 0.333 & & $x_{2}$ & 0.333 & 0.333 & 0.333 \\
$x_{3}$ & 0.222 & 0.222 & 0.556 & & $x_{3}$ & 0.056 & 0.889 & 0.056 \\
$x_{4}$ & 0.444 & 0.278 & 0.278 & & $x_{4}$ & 0.238 & 0.222 & 0.556
\end{tabular}

\subsection{Coincidence matrix}

We adopted the definition of fuzzy coincidence by (Herrera et al. 1997, p.

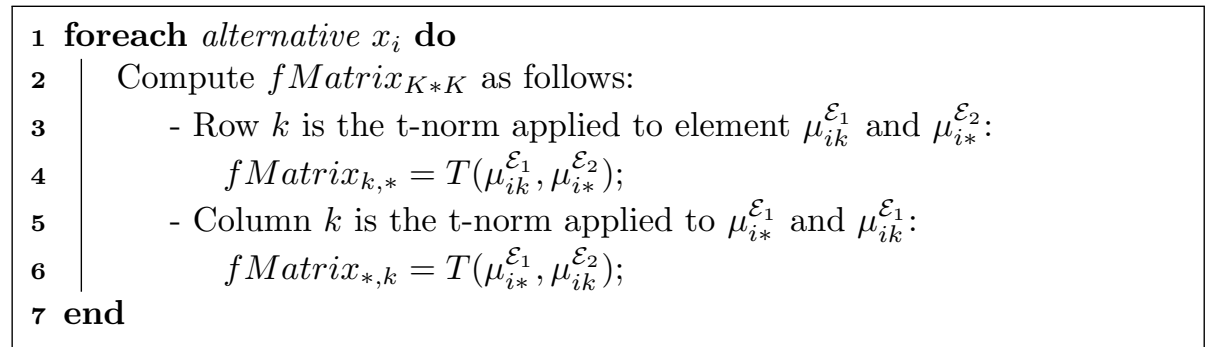

Algorithm 3: Computing coincidence matrix for t-norm

Example 3. Let us compute the coincidence matrix of the previous example assuming a product t-norm. Table 5 depicts the values obtained for the coincidence matrix. 
Table 5: Example of coincidence matrix between experts $\mathcal{E}_{1}$ and $\mathcal{E}_{2}$ regarding alternative $x_{1}$

\begin{tabular}{|c|c|c|c|c|c|c|c|c|}
\hline & A & B & $\mathrm{C}$ & & & A & B & $\mathrm{C}$ \\
\hline A & $0.667 \cdot 0.889$ & $0.667 \cdot 0.056$ & $0.667 \cdot 0.056$ & & A & 0.593 & 0.037 & 0.037 \\
\hline B & $0.167 \cdot 0.889$ & $0.167 \cdot 0.056$ & $0.167 \cdot 0.056$ & $=$ & B & 0.148 & 0.009 & 0.009 \\
\hline C & $0.167 \cdot 0.889$ & $0.167 \cdot 0.056$ & $0.167 \cdot 0.056$ & & $\mathrm{C}$ & 0.148 & 0.009 & 0.009 \\
\hline
\end{tabular}

\subsection{Soft consensus degree} 1997; Herrera-Viedma et al., 2014). Our proposed measure is based on the fuzzy kappa index introduced in Subsection 2.4. It adds robustness since it takes into account agreement occurring by chance. Algorithm 4 details the computation of fuzzy kappa index implemented according to the definitions given by Dou et al.

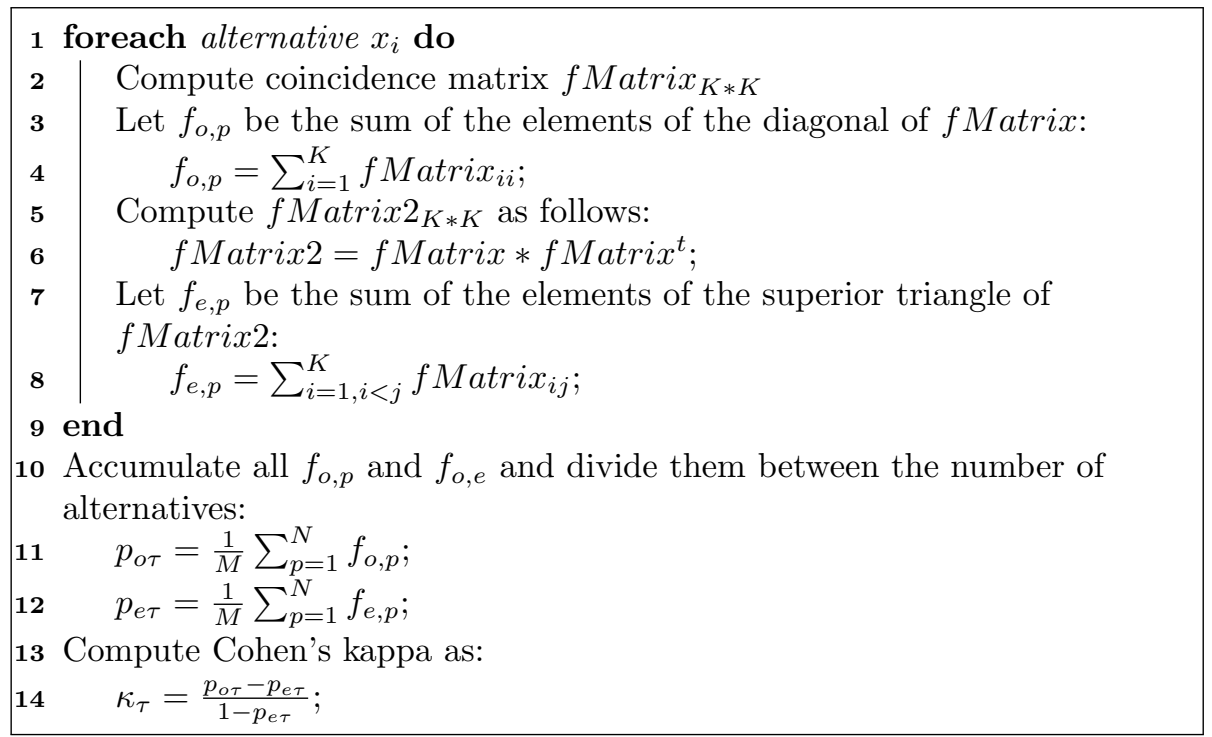

Algorithm 4: Computing fuzzy kappa

Example 4. To conclude with the previous example, let us compute $\kappa_{\tau}$ following Algorithm 4. The first step is to compute the partial $f_{o, p}$ and $f_{e, p}$ $f_{o, 1}=0.593+0.009+0.009=0.612, f_{e, 1}=0.037 \cdot 0.148+0.037 \cdot 0.148+0.009$. $0.009=0.011, f_{o, 2}=0.333, f_{e, 2}=0.037, f_{o, 3}=0.241, f_{e, 3}=0.009, f_{o, 4}=0.322$ 
330

0.022 and observed probability $p_{o \tau}=\frac{0.612+0.333+0.241+0.322}{4}=0.377$ are then computed. Finally, fuzzy kappa or the soft consensus degree is obtained $\kappa_{\tau}=$ $\frac{0.377-0.022}{1-0.022}=0.363$.

\subsection{Extending soft consensus degree to several decision-makers}

In this subsection, a extension of the soft consensus degree from two to several decision-makers who each assign a set of alternatives into several categories by means of a fuzzy partition is proposed. Herrera-Viedma et al. (2014) identify two approaches to measure agreement for three or more decision-makers according to reference domain (i.e. decision-makers or alternatives). The first type Carlsson et al. 1992), while the second type is based on a fuzzy partition per alternative (Alonso et al., 2013, Cabrerizo et al., 2010, Palomares et al., 2014). This approach can be applied to our proposed two-decision-maker soft consensus degree (as shown below) since the index introduced in (2) takes into account hen, 1968). Thus, we consider a decision-maker-based approach to extend the considered consensus degree described in three steps:

Step 1: Soft consensus degree between a pair of decision-makers, $i$ and $k$ : $c d e_{i k}=\kappa_{\tau}$.

350 Step 2: Average of consensus degrees for each decision-maker: $c a_{i}=\sum_{k=1, k \neq i}^{R} \frac{c d e_{i k}}{R-1}$, where $R$ stands for the number of decision-makers.

Step 3: Aggregated soft consensus degree for the group of decision-makers: $c g d=\sum_{i=1}^{R} \frac{c a_{i}}{R}$.

Most real-life situations measuring consensus are based on the preferences of several decision-makers, instead of two decision-makers' preferences. This extension allows us to measure consensus degree by taking into account the different categories' occurrences. 


\section{Use Case in Innovation Contest}

\subsection{Problem definition}

\subsection{Data}

Data was obtained from an innovation contest operated by the United States Agency for International Development (USAID), a U.S. federal agency for funding developing countries. USAID offered to the development public the opportunity to suggest development programme ideas using a 3-day innovation contest event. There were several different topics that people could focus on, including, for example, empowering women. This event was promoted by partnering with several organisations throughout the world, and 1279 individuals from 135 countries participated, generating 5268 posts across five topics.

A real-life experiment, in which the posts from five topics were categorised using the proposed methodology based on one confidence degree per post, was conducted. A jury of staff members from the USAID strategy unit synthesised 

average number of words.

Table 6: Statistics of the posts and contests

\begin{tabular}{|c|c|c|c|c|c|}
\hline Contest & $\begin{array}{l}\text { \# Posts per } \\
\text { contest }\end{array}$ & Ideas & Batch & $\begin{array}{c}\text { \# Posts per } \\
\text { batch }\end{array}$ & $\begin{array}{c}\text { \# Words per } \\
\text { post }\end{array}$ \\
\hline \multirow{4}{*}{$\begin{array}{l}\text { Empowering Women } \\
\text { and Girls }\end{array}$} & \multirow{4}{*}{1618} & \multirow{4}{*}{7} & 1 & 100 & 106 \\
\hline & & & 2 & 200 & 134 \\
\hline & & & 3 & 300 & 142 \\
\hline & & & 4 & 1018 & 120 \\
\hline Pursuing & \multirow{3}{*}{591} & \multirow{3}{*}{4} & 1 & 100 & 215 \\
\hline Grand & & & 2 & 200 & 160 \\
\hline Challenges & & & 3 & 291 & 137 \\
\hline Fostering Science, & \multirow{3}{*}{768} & \multirow{3}{*}{6} & 1 & 100 & 119 \\
\hline Technology and & & & 2 & 200 & 112 \\
\hline Innovation & & & 3 & 468 & 115 \\
\hline \multirow{4}{*}{$\begin{array}{l}\text { Inspiring a } \\
\text { New Generation }\end{array}$} & \multirow{4}{*}{1515} & \multirow{4}{*}{6} & 1 & 100 & 136 \\
\hline & & & 2 & 200 & 122 \\
\hline & & & 3 & 300 & 150 \\
\hline & & & 4 & 915 & 126 \\
\hline Building & \multirow{3}{*}{776} & \multirow{3}{*}{6} & 1 & 100 & 176 \\
\hline Stronger & & & 2 & 200 & 162 \\
\hline Partnerships & & & 3 & 476 & 142 \\
\hline
\end{tabular}




\subsection{Results}

Each decision-maker assessed the idea and level of confidence for each post for a topic. Three different ways of measuring agreement were computed to assess the effect of possible decisions on the fuzzification process using product t-norm for the fuzzy kappa (see (3p). Moreover, crisp kappa was calculated in order to have a threshold measure. Table 7 details the results obtained.

Table 7: Consensus degrees using the product t-norm

\begin{tabular}{cccccc} 
Contest & Batch & Crisp & Linear & Logarithmic & Exponential \\
\hline \hline Building stronger & 2 & 0.32 & 0.67 & 0.74 & 0.51 \\
partnerships & 3 & 0.26 & 0.79 & 0.82 & 0.68 \\
\hline Empowering & 2 & 0.46 & 0.74 & 0.84 & 0.34 \\
women and girls & 3 & 0.45 & 0.75 & 0.85 & 0.37 \\
& 4 & 0.80 & 0.79 & 0.87 & 0.51 \\
\hline Fostering, science, & 2 & 0.23 & 0.82 & 0.86 & 0.67 \\
technology, and science & 3 & 0.46 & 0.82 & 0.86 & 0.66 \\
\hline Pursuing & 2 & 0.42 & 0.79 & 0.85 & 0.56 \\
major challenges & 3 & 0.39 & 0.73 & 0.80 & 0.48
\end{tabular}

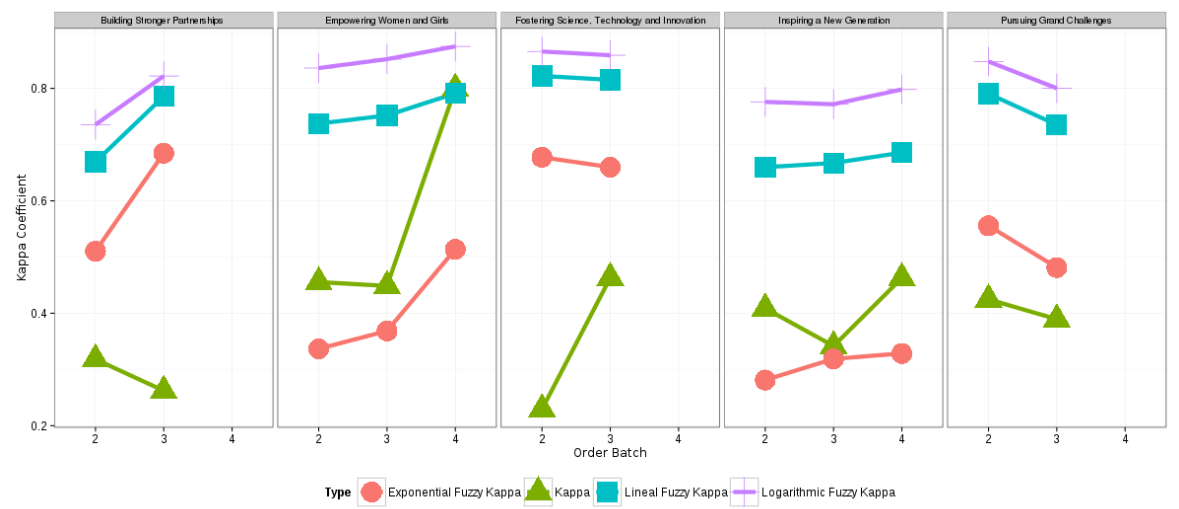

Figure 4: Agreement measurement by type of kappa and innovation contest using probabilistic product as the t-norm

Figure 4 shows that, despite using product t-norm, kappa's estimation order from each different fuzzification normalisation function (i.e. linear, exponen- 
tial, and logarithmic) is as expected. Two overconfident decision-makers show more agreement than an average underconfident decision-maker when using the same confidence degrees. An exponential fuzzification is more accurate than the traditional crisp kappa and other considered fuzzy kappa definitions (i.e. logarithmic and linear).

Consensus is clearly improving over time in all but the smaller contests (i.e. "Pursuing major challenges"). This indicates that the level of agreement improved over time as long as the data set is large enough (>600 posts). The use of confidence degrees improved the quality and efficiency of the experts' categorisation by decreasing time for each consecutive batch (as reported one expert). A possible explanation for this decrease in time is that experts do not need to be absolutely certain about their decisions since humans have their own subjectivity (Herrera-Viedma et al. 2014). This proposed method enabled us to capture this uncertainty at a reasonable cost (i.e. confidence degree for patterns instead of categories). Moreover, this confidence degree was offered as a feedback mechanism for those patterns where agreement was not reached, and so providing additional support for the discussion.

\section{Discussion}

This paper proposes a feasible method to consider the fuzzy nature of decisions when categorising a set of alternatives by means of confidence degrees. The method enables the use of different fuzzyfication functions - three of which are depicted in Table 3 - and the use of different t-norms described in Section 2.2 The selection of these elements has several implications that are discussed. Linear, exponential, and logarithmic functions are considered when associated respectively with normal, underconfident, and overconfident decision-makers. Overconfident decision-makers, whose subjective confidence in their judgement is reliably greater than objective judgement (Pallier et al., 2002), are compensated by using a logarithmic normalisation function during the fuzzification process. Underconfident people, whose subjective confidence in their judgement is 
reliably lower than the objective judgement, are compensated by using an exponential normalisation function. Finally, people with normal confidence, whose subjective confidence is close to the objective function, are not compensated using a linear normalisation function.

The selection of the t-norm to obtain the shared membership degrees (i.e. coincidence matrix) is relevant. A minimum t-norm, that represents the standard semantics for weak conjunction $\operatorname{logic} T(x, y)=\min (x, y)$, overemphasises total agreements among the two decision-makers, and thus reduces the relevance of disagreements. A product t-norm (i.e. $T(x, y)=x \cdot y$ ) penalises low partial agreement, while boosting high partial agreement. A Lukasiewicz t-norm, that is pointwise smaller than the product t-norm $T(x, y)=\max \{x+y-1,0\}$, overpenalises low partial agreement while overboosting high partial agreement.

Several measures exist to assess the level of agreement, ranging from simple ratio agreements to measures that take into account the distribution of each category (Cohen, 1968). The consensus degree defined in this paper introduces ${ }_{45}$ the use of a measure that takes into account the distribution of each category that is fuzzy by nature (Dou et al. 2007). Table 8 compares the typical consensus forms of measurement in three dimensions: 1) implementation complexity; 2 ) assumption of the distribution of categories; and 3) certainty of the decisionmaker at each decision. The ratio measurement considers that all categories are equally likely, hence introducing distortion in the measurement. The kappa improves ratio measurement by considering that each category is not equally likely to happen. Finally, fuzzy kappa considers that every idea or category is not equally likely to happen and that the decision-makers are not always certain about their decisions.

\section{Conclusions and Future Research}

The focus of this research was to develop a fuzzy measure of agreement through fuzzy kappa based on fuzzy partitions that consider the fuzzy nature of GDM using one confidence degree for each decision (instead of for each cat- 
Table 8: Comparison of consensus degrees

\begin{tabular}{lclll} 
Measure & Formula & Complexity & Distribution of categories & Certainty decision \\
\hline \hline Ratio & $P_{o}^{i k}$ & Simple & Equally likely & Constant \\
\hline kappa & $\kappa=\frac{p_{o}-p_{e}}{1-p_{e}}$ & Medium & $\begin{array}{l}\text { Some categories are more } \\
\text { likely than others }\end{array}$ & Constant \\
\hline Fuzzy kappa & $\kappa_{\tau}=\frac{p_{o \tau}-p_{e \tau}}{1-p_{e \tau}}$ & Advanced & $\begin{array}{l}\text { Some categories are more } \\
\text { likely than others }\end{array}$ & Not constant \\
\hline
\end{tabular}

egory). This confidence degree is used to define a fuzzy partition based on decision-maker preferences. A coincidence matrix is computed by two or more fuzzy partitions using a specific t-norm. Finally, the consensus degree is obtained from this coincidence matrix.

A real use case in an innovation contest is considered. The results suggest that this methodology captures the degree of consensus more accurately, without much additional effort. Moreover, the fuzzification of the decision allows us to model and normalise different types of confidence (i.e. overconfidence, average confidence and underconfidence). Finally, the assessment of the different conceptualisations of t-norm enables us to consider less strict coincidence models (i.e. probabilistic product t-norm) or stricter models (i.e. min t-norm).

The limitations of this research are related to the constraints imposed on using real-life experiments to assess different parametrisation (i.e. fuzzification parametrisation and different types of t-norm for the fuzzy kappa). Thus, future research should estimate acceptable and optimal fuzzy consensus degrees for the different parametrisations of the kappa index.

\section{Acknowledgments}

This work was partially supported by the SENSORIAL Research Project (TIN2010-20966-C02-01, 02), funded by the Spanish Ministry of Science and Information Technology. 
The authors would like to thank NSF 11 for their generous funding of this research (NSF \#1219832). Three research assistants contributed by categorising the data in this paper: Sabrina Briscoe, Eric Brown, and José Gómez. Lastly, the authors would like to thank Dr. Esteve Almirall for providing access to the innovation contest.

\section{References}

${ }_{485}$ Administration, U.G.S., 2015. Challenge.org. URL: http://www.challenge. gov,

Alonso, S., Pérez, I.J., Cabrerizo, F.J., Herrera-Viedma, E., 2013. A linguistic consensus model for web 2.0 communities. Applied Soft Computing 13, 149157.

490 Ayad, H.G., Kamel, M.S., 2010. On voting-based consensus of cluster ensembles. Pattern Recognition 43, 1943-1953.

Bellman, R.E., Zadeh, L.A., 1970. Decision-making in a fuzzy environment. Management science 17, B-141.

Bressen, T., 2007. Consensus decision making, in: The Change Handbook: The Definitive Resource on Today's Best Methods for Engaging Whole Systems (2nd. Ed.). Berrett-Koehler Publishers, San Francisco, CA, pp. 212-217.

Bullinger, A.C., Neyer, A.K., Rass, M., Moeslein, K.M., 2010. Communitybased innovation contests: Where competition meets cooperation. Creativity and innovation management 19, 290-303.

500

Cabrerizo, F.J., Alonso, S., Herrera-Viedma, E., 2009. A consensus model for group decision making problems with unbalanced fuzzy linguistic information. International Journal of Information Technology \& Decision Making 8, 109131.

\footnotetext{
${ }^{1}$ NSF: National Science Foundation
} 
Cabrerizo, F.J., Moreno, J.M., Pérez, I.J., Herrera-Viedma, E., 2010. Analyz-

ing consensus approaches in fuzzy group decision making: advantages and drawbacks. Soft Computing 14, 451-463.

Campello, R.J., 2007. A fuzzy extension of the rand index and other related indexes for clustering and classification assessment. Pattern Recognition Letters $28,833-841$.

Carlsson, C., Ehrenberg, D., Eklund, P., Fedrizzi, M., Gustafsson, P., Lindholm, P., Merkuryeva, G., Riissanen, T., Ventre, A.G., 1992. Consensus in distributed soft environments. European Journal of Operational Research 61, $165-185$.

Chen, C.T., 2000. Extensions of the topsis for group decision-making under fuzzy environment. Fuzzy sets and systems 114, 1-9.

Chesbrough, H., 2006. Open innovation: A new paradigm for understanding industrial innovation, in: Chesbrough, H., Vanhaverbeke, W., West, J. (Eds.), Open innovation: Researching a new paradigm. Oxford university press, pp. $1-25$.

Chiclana, F., Tapia García, J., del Moral, M.J., Herrera-Viedma, E., 2013. A statistical comparative study of different similarity measures of consensus in group decision making. Information Sciences 221, 110-123.

Cohen, J., 1968. Weighted kappa: Nominal scale agreement provision for scaled disagreement or partial credit. Psychological bulletin 70, 213.

${ }_{525}$ Cooper, R.G., De Brentani, U., 1984. Criteria for screening new industrial products. Industrial Marketing Management 13, 149-156.

Diehl, M., Stroebe, W., 1987. Productivity loss in brainstorming groups: Toward the solution of a riddle. Journal of personality and social psychology $53,497$. 
Dou, W., Ren, Y., Wu, Q., Ruan, S., Chen, Y., Bloyet, D., Constans, J.M., 2007. Fuzzy kappa for the agreement measure of fuzzy classifications. Neurocomputing $70,726-734$.

Dubois, D., Prade, H., 2004. On the use of aggregation operations in information fusion processes. Fuzzy Sets and Systems 142, 143-161.

Edwards, W., 1977. How to use multiattribute utility measurement for social decisionmaking. Systems, Man and Cybernetics, IEEE Transactions on 7, 326-340.

Ferguson, D., 2010. Global Pulse 2010: Insights and ideas from around the world. Technical Report. United States Agency for International Development.

Fowlkes, E.B., Mallows, C.L., 1983. A method for comparing two hierarchical clusterings. Journal of the American statistical association 78, 553-569.

Füller, J., Hutter, K., Fries, M., 2012. Crowdsourcing for goodness sake: Impact of incentive preference on contribution behavior for social innovation. Adv. Int. Market 23, 137-159.

Gwet, K.L., 2014. Handbook of inter-rater reliability: The definitive guide to measuring the extent of agreement among raters. Advanced Analytics, LLC.

Halkidi, M., Batistakis, Y., Vazirgiannis, M., 2001. On clustering validation techniques. Journal of Intelligent Information Systems 17, 107-145.

Herrera, F., Herrera-Viedma, E., Verdegay, J.L., 1997. Linguistic measures based on fuzzy coincidence for reaching consensus in group decision making. International Journal of Approximate Reasoning 16, 309-334.

Herrera-Viedma, E., Alonso, S., Chiclana, F., Herrera, F., 2007. A consensus model for group decision making with incomplete fuzzy preference relations. Fuzzy Systems, IEEE Transactions on 15, 863-877. 
Herrera-Viedma, E., Cabrerizo, F.J., Kacprzyk, J., Pedrycz, W., 2014. A review of soft consensus models in a fuzzy environment. Information Fusion 17, 4-13.

Herrera-Viedma, E., Herrera, F., Chiclana, F., 2002. A consensus model for multiperson decision making with different preference structures. Systems, Man and Cybernetics, Part A: Systems and Humans, IEEE Transactions on $32,394-402$.

Herrera-Viedma, E., Martinez, L., Mata, F., Chiclana, F., 2005. A consensus support system model for group decision-making problems with multigranular linguistic preference relations. Fuzzy Systems, IEEE Transactions on 13, 644658.

Hubert, L., Arabie, P., 1985. Comparing partitions. Journal of classification 2, $193-218$.

Jain, A.K., Dubes, R.C., et al., 1988. Algorithms for clustering data. volume 6. Prentice hall Englewood Cliffs.

570 Jiang, D., Tang, C., Zhang, A., 2004. Cluster analysis for gene expression data: A survey. Knowledge and Data Engineering, IEEE Transactions on 16, $1370-1386$.

Kacprzyk, J., Fedrizzi, M., 1988. A softmeasure of consensus in the setting of partial (fuzzy) preferences. European Journal of Operational Research 34, $575 \quad 316-325$.

Kaner, S., 2014. Facilitator's guide to participatory decision-making. John Wiley \& Sons.

King, A.A., Lakhani, K.R., 2011. The contingent effect of absorptive capacity: An open innovation analysis. Harvard Business School.

580 Klink, R.R., Athaide, G.A., 2006. An illustration of potential sources of concepttest error*. Journal of Product Innovation Management 23, 359-370. 
Kukar, M., 2003. Transductive reliability estimation for medical diagnosis. Artificial Intelligence in Medicine 29, 81-106.

Lamastra, C.R., 2009. Software innovativeness. a comparison between proprietary and free/open source solutions offered by italian smes. R\&D Management 39, 153-169.

Landis, J.R., Koch, G.G., 1977. The measurement of observer agreement for categorical data. biometrics , 159-174.

Leimeister, J.M., Huber, M., Bretschneider, U., Krcmar, H., 2009. Leveraging crowdsourcing: activation-supporting components for it-based ideas competition. Journal of management information systems 26, 197-224.

Linstone, H.A., Turoff, M., et al., 1975. The Delphi method: Techniques and applications. volume 29. Addison-Wesley Reading, MA.

Majchrzak, A., Malhotra, A., 2013. Towards an information systems perspective and research agenda on crowdsourcing for innovation. The Journal of Strategic Information Systems 22, 257-268.

Malhotra, A., Majchrzak, A., 2014. Managing crowds in innovation challenges. California Management Review 56.

Martínez, L., Montero, J., 2007. Challenges for improving consensus reaching process in collective decisions. New Mathematics and Natural Computation $3,203-217$.

Neyer, A.K., Bullinger, A.C., Moeslein, K.M., 2009. Integrating inside and outside innovators: a sociotechnical systems perspective. R\&d Management 39, 410-419.

OpenIDEO, 2015. OpenIDEO - Where people design better, together. URL: https://openideo.com/

Osei-Bryson, K.M., 2010. Towards supporting expert evaluation of clustering results using a data mining process model. Information Sciences 180, 414-431. 
Pallier, G., Wilkinson, R., Danthiir, V., Kleitman, S., Knezevic, G., Stankov, L., Roberts, R.D., 2002. The role of individual differences in the accuracy of confidence judgments. The Journal of General Psychology 129, 257-299.

Palomares, I., Estrella, F.J., Martínez, L., Herrera, F., 2014. Consensus under a fuzzy context: Taxonomy, analysis framework afryca and experimental case of study. Information Fusion 20, 252-271.

Piller, F.T., Walcher, D., 2006. Toolkits for idea competitions: a novel method to integrate users in new product development. r\&D management $36,307-$ 318.

Rand, W.M., 1971. Objective criteria for the evaluation of clustering methods. Journal of the American Statistical association 66, 846-850.

of the Red Cross, I.C., 2015. How Does co-creation Change the Approach People

口 are Taking to Innovaiton? URL: http://blogs.icrc.org/gphi2/2014/12/ 19/co-creation/.

Ruspini, E.H., 1969. A new approach to clustering. Information and control 15, $22-32$.

Saldaña, J., 2012. The coding manual for qualitative researchers. 14, Sage.

Santos, R., Spann, M., 2011. Collective entrepreneurship at qualcomm: combining collective and entrepreneurial practices to turn employee ideas into action. R\&D Management 41, 443-456.

Simonton, D.K., 1999. Origins of genius: Darwinian perspectives on creativity. 630 Oxford University Press.

Surowiecki, J., 2005. The wisdom of crowds. Anchor.

Torrance, G.W., Boyle, M.H., Horwood, S.P., 1982. Application of multiattribute utility theory to measure social preferences for health states. Operations research 30, 1043-1069. 
Toubia, O., 2006. Idea generation, creativity, and incentives. Marketing Science $25,411-425$.

Von Hippel, E., 1989. Cooperation between rivals: informal know-how trading. Springer.

Zadeh, L.A., 1965. Fuzzy sets. Information and control 8, 338-353. 
Graphical Abstract

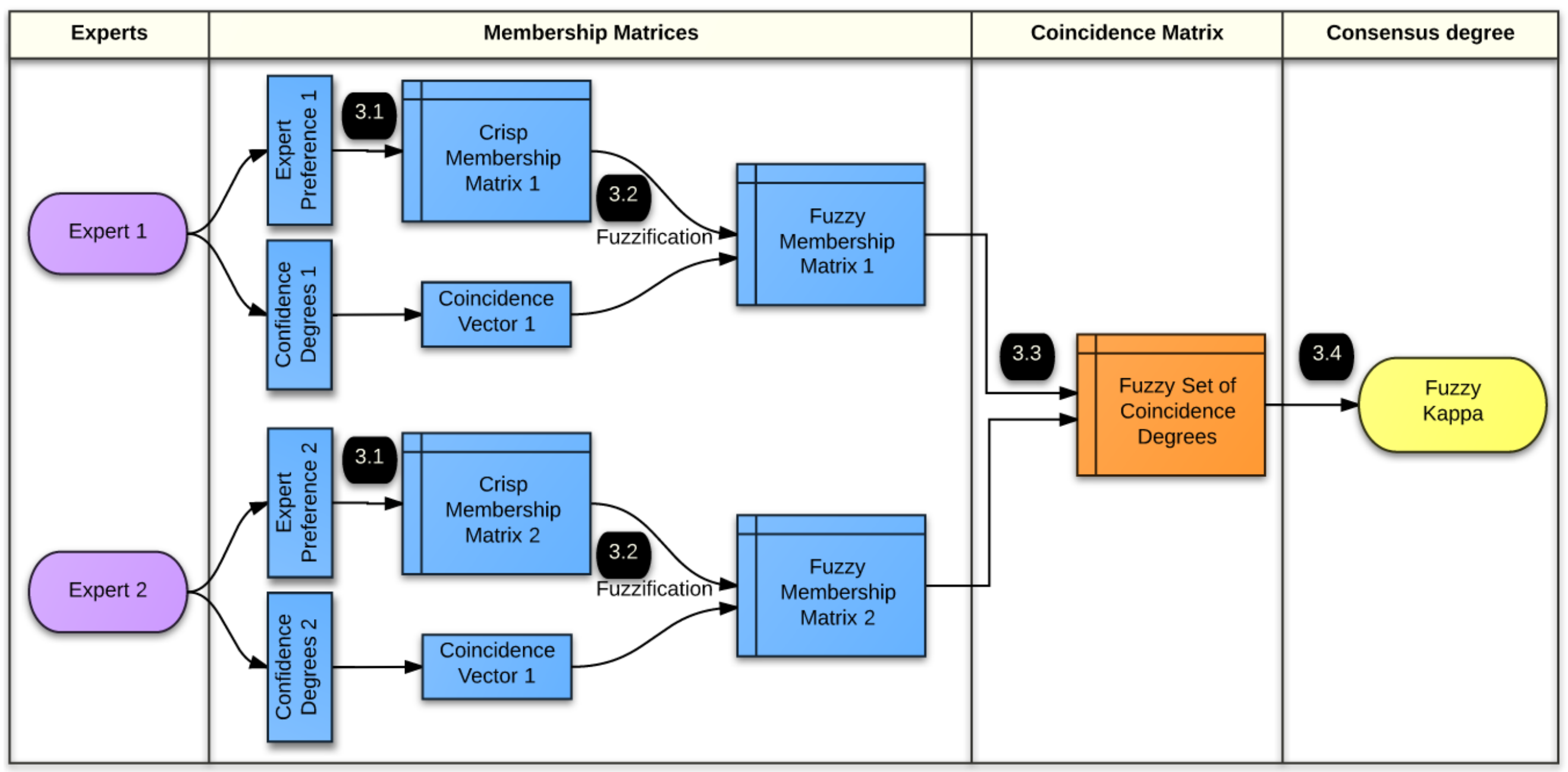

